\title{
Autosomal dominant proximal renal tubular acidosis
}

INSERM

\section{Source}

INSERM. (1999). Orphanet: an online rare disease and orphan drug data base. Autosomal dominant proximal renal tubular acidosis. ORPHA:314889

Autosomal dominant proximal renal tubular acidosis (AD pRTA) is a form of proximal renal tubular acidosis (pRTA; see this term) characterized by an isolated defect in the proximal tubule leading to the decreased reabsorption of bicarbonate and consequently causing urinary bicarbonate wastage. Mild growth retardation and reduced bone density are extra-renal complications. 